\title{
Acute mechanical stress in primary porcine RPE cells induces angiogenic factor expression and in vitro angiogenesis
}

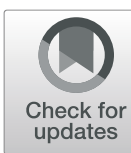

\author{
Farhad Farjood ${ }^{1,2}$ (D) Amir Ahmadpour ${ }^{1,3}$ (D), Sassan $\operatorname{Ostvar}^{4}(\mathbb{D})$ and Elizabeth Vargis $^{1 *}$ (D)
}

\begin{abstract}
Background: Choroidal neovascularization (CNV) is a major cause of blindness in patients with age-related macular degeneration. CNV is characterized by new blood vessel growth and subretinal fluid accumulation, which results in mechanical pressure on retinal pigment epithelial (RPE) cells. The overexpression of RPE-derived angiogenic factors plays an important role in inducing CNV. In this work, we investigated the effect of mechanical stress on the expression of angiogenic factors in porcine RPE cells and determined the impact of conditioned medium on in-vitro angiogenesis.

Results: The goal of this study was to determine whether low levels of acute mechanical stress during early CNV can induce the expression of angiogenic factors in RPE cells and accelerate angiogenesis. Using a novel device, acute mechanical stress was applied to primary porcine RPE cells and the resulting changes in the expression of major angiogenic factors, VEGF, ANG2, HIF-1a, IL6, IL8 and TNF-a, were examined using immunocytochemistry, qRT-PCR, and ELISA. An in vitro tube formation assay was used to determine the effect of secreted angiogenic proteins due to mechanical stress on endothelial tube formation by human umbilical vein endothelial cells (HUVECs). Our results showed an increase in the expression of VEGF, ANG2, IL-6 and IL-8 in response to mechanical stress, resulting in increased in vitro angiogenesis. Abnormal epithelial-mesenchymal transition (EMT) in RPE cells is also associated with CNV and further retinal degeneration. Our qRT-PCR results verified an increase in the expression of EMT genes, $C D H 2$, VIM and FN1, in RPE cells.
\end{abstract}

Conclusions: In conclusion, we showed that acute mechanical stress induces the expression of major angiogenic and EMT factors and promotes in vitro angiogenesis, suggesting that mechanical stress plays a role in promoting aberrant angiogenesis in AMD.

Keywords: Mechanical stress, Angiogenesis, RPE, AMD, EMT, CNV, VEGF, IL-6, IL-8, ANG2

\section{Background}

The choroid is a vascular layer underneath the retinal pigment epithelium (RPE) that supplies blood to the RPE and the retina. The abnormal overgrowth of choroidal blood vessels creates a condition called choroidal

\footnotetext{
*Correspondence: vargis@usu.edu

'Department of Biological Engineering, Utah State University, 4105 Old Main Hill, Logan, UT 84322, USA

Full list of author information is available at the end of the article
}

neovascularization (CNV). In age-related macular degeneration (AMD), CMV damages the overlying RPE and photoreceptors, resulting in irreversible blindness. The etiology of CNV remains to be fully outlined, but RPE cells do produce higher levels of angiogenic proteins in response to mechanical stress, promoting angiogenesis and contributing to CNV development $[1,2]$. Choroidal blood vessel invasion and sub-RPE fluid accumulation are potential sources of mechanical stress during AMD. As new blood vessels form, creating spatial crowding and hemorrhages, RPE cells elongate from $<10 \%$ to 
$60 \%$, uniaxially [3-5]. However, little is known about the resulting angiogenic factor expression in RPE cells.

Many pro-angiogenic proteins are involved in $\mathrm{CNV}$, including the RPE-derived vascular endothelial growth factor (VEGF), angiopoietin 2 (ANG2), and fibroblast growth factor 2 (FGF2) [6-8]. Interleukin-6 (IL-6), interleukin-8 (IL-8) and tumor necrosis factor- $\alpha$ (TNF$\alpha)$, are also involved in choroidal angiogenesis [9-11]. Another marker of CNV pathogenesis is epithelialmesenchymal transition (EMT), which promotes RPE de-differentiation $[12,13]$, and is triggered by angiogenic cytokines, such as TNF- $\alpha$, VEGF, Il-6 and IL-8 [14-17]. Mechanical stress may promote EMT in RPE cells by inducing the expression of these factors.

To test whether increased mechanical stress on RPE cells during early stages of CNV accelerates CNV development, we modified an in vitro technique [2] to model low levels of strain in the RPE (10\% uniaxial strain) to mimic those experienced during early CNV. Then, we studied how mechanical stress effects mRNA and protein expression of major angiogenic factors: VEGF, ANG2, hypoxia-inducible factor- $1 \alpha$ (HIF- $1 \alpha)$, IL-6, IL-8, and TNF- $\alpha$; and EMT markers: vimentin (VIM), cadherin 2 (CDH2), and fibronectin-1 (FN1). In addition, we used finite element analysis and immunocytochemistry to find correlations between strain levels and increased expression of VEGF, IL-6 and IL-8. We also assessed the angiogenic potential of the stressinduced RPE secretome using an in vitro angiogenesis assay with human umbilical vein endothelial cells (HUVECs). HUVECs are capable of forming endothelial tube-like networks, similar to microvasculature, when cultured on 3D basement membranes and, therefore, have been widely used to study angiogenesis in different experimental settings $[18,19]$. We employed HUVECs to evaluate the angiogenesis potential of the conditioned media from RPE cultures on in vitro angiogenesis. Our results showed that mechanical stress similar to that experienced during early CNV induces the expression of angiogenic and EMT factors in RPE cells, further promoting in vitro angiogenesis.

\section{Results}

\section{Characterization of isolated porcine RPE cells}

Isolated porcine RPE cells showed characteristics of differentiated RPE after 4 weeks of culture on Transwell membranes. Confocal images showed adherens junction protein, $\beta$-catenin (Fig. 2a), F-actin (Fig. 2b), and tight junction protein, ZO-1 (Fig. 2c) along the cell-cell junctions, as well as the cytoplasmic expression of the RPEspecific protein, RPE65 (Fig. 2d). The typical RPE cobblestone morphology was also observed in brightfield images of 4-week-old cultures (Fig. 2e). The TEER values increased from 65.38 to $767.05 \Omega . \mathrm{cm}^{2}(p=1.46 \mathrm{E}$ 6) after 4 weeks (Fig. 2f).

\section{Increased strain leads to increased VEGF, IL-6, and IL-8 expression}

Finite element analysis showed that the mechanically stressed area's center experiences the highest strain level (Fig. 3a). These results were compared to the ICC results to determine whether strain levels and increased angiogenic protein expression are correlated.

Confocal images of mechanically-stressed RPE cells showed that in non-stressed cells, F-actin localized to cell-cell junctions (Fig. 2b), while in mechanically stressed cells, the actin filaments distributed diffusely in the cytoplasm (Fig. 3c, h, m). ICC results also showed that the mechanical stress-induced disruption of the Factin cytoskeleton was associated with increases in VEGF (Fig. 3b-e), IL-6 (Fig. 3g-j), and IL-8 (Fig. 3l-o) expression. The RPE monolayer also began to deform after stretching the Transwell membranes, based on z-stack images and (Fig. 3f, k, p), as predicted by FEA (Fig. 3a).

\section{Mechanical stress in RPE cells induces the expression of angiogenic and EMT factors and promotes in vitro angiogenesis}

qRT-PCR results showed substantial changes in the expression of major angiogenic and EMT mRNA (Fig. 3q, r). Three hours after applying mechanical stress, the expression of angiogenic factors, VEGF121, HIF-1 $\alpha, A N G 2$ and $I L-8$, increased significantly $(p=0.018,0.007,0.021,0.018$, respectively). The expression of pigment epitheliumderived factor (PEDF), an anti-angiogenic gene, was also increased $(p=0.044)$. We observed a significant increase in the expression of the EMT promoter, TNF- $\alpha$ ( $p=$ 0.005). After 6 hours, the expression of two major VEGF isoforms, VEGF121 and VEGF165, and a stimulator of VEGF expression, HIF- $1 \alpha$, increased $(p=0.011,0.016$, 0.011, respectively). Moreover, the expression of ANG2, $I L-6$ and $I L-8, P E D F$ and TNF- $\alpha$ also increased significantly ( $p=0.017,0.011,0.013,0.013,0.016$, respectively; Fig. 3q). While FGF2 gene expression in mechanically stressed RPE cells decreased significantly after 6 hours $(p=0.017)$, there was no significant change in apical and basal FGF2 protein expression $(p=0.52,0.49$, respectively; Fig. 3q, s, t).

We also observed a significant increase in the following EMT markers, CDH2 (p $(3 \mathrm{~h})=0.044, \mathrm{p} \quad(6 \mathrm{~h})=$ 0.024), VIM (p $(3 \mathrm{~h})=0.094, \mathrm{p}(6 \mathrm{~h})=0.017)$, and FN1 (p $(3 \mathrm{~h})=0.044, \mathrm{p}(6 \mathrm{~h})=0.017)$, and a decrease in CDH1 $(\mathrm{p}$ $(3 \mathrm{~h})=0.044, \mathrm{p}(6 \mathrm{~h})=0.051)$ and an RPE-specific gene, RPE65 (p $(3 \mathrm{~h})=0.03, \mathrm{p}(6 \mathrm{~h})=0.00$; Fig. 3r). ELISA results showed a significant increase in the expression of IL-6, apically ( $p=0.014$ respectively), and VEGF, ANG2, 
IL-6 and IL-8, basolaterally ( $p=0.042,0.008,0.000$, 0.019 respectively; Fig. 3s, t).

qRT-PCR results showed a concurrent increase in proangiogenic gene expression, as mentioned above, as well as the anti-angiogenic factor, PEDF (Fig. 3q). To determine whether the increase of pro-angiogenic factors overrides the anti-angiogenic activity of PEDF, an in vitro angiogenesis assay was performed. Conditioned media from RPE cultures activated endothelial tube formation, while limited angiogenic activity was observed in HUVECs cultured in fresh RPE media (Fig. 4). Exposure to apical and basal conditioned media from mechanically stressed RPE cells resulted in a significant $\sim 2$ - and 1.4-fold increase in endothelial tube length $(p=0.001$, 0.017 , respectively) and $\sim 3.6$ - and 2-fold increase in node number respectively $(p=0.003,0.018$, respectively; Fig. 4f, g).

\section{Discussion}

While the mechanisms leading to increased expression of RPE-derived angiogenic factors and the resulting angiogenesis during $\mathrm{CNV}$ in AMD are not entirely clear, physical changes in the RPE contribute to the elevated expression of angiogenic factors $[1,2,20]$. To better understand the role that mechanical stress plays in AMD pathogenesis, we investigated the effect of mechanical stress on the expression of angiogenic and EMT factors and in vitro angiogenesis using a novel in vitro device (Fig. 1).

Mechanical stress in many cell types, including periodontal ligament fibroblasts (HPLF), mesenchymal stem cells and HUVECs, has been found to affect angiogenesis [21-23]. ARPE-19 cells, a human RPE cell line, express increased levels of angiogenic cytokines, such as VEGF and IL6, involved in various retinal diseases in response to cyclic mechanical stress [1, 24]. However, ARPE-19 cells lack important RPE characteristics, such as RPEspecific marker expression and high transepithelial resistance [25]. A more realistic RPE model is needed to characterize the effect of mechanical stress-derived changes to the RPE secretome and angiogenesis. To this end, we produced a realistic in vitro model of the RPE by growing freshly isolated porcine RPE cells on Transwell membranes. Porcine RPE has been used previously as a realistic in vitro model to study many RPE functions, including VEGF expression and in vitro angiogenesis, demonstrating its suitability for the purpose of this study [26-28]. Our in vitro porcine RPE cultures demonstrated several characteristics similar to native RPE, such as junctional localization of $\beta$-catenin, $F$-actin, and ZO-1, expression of the RPE-specific RPE65, and high TEER in 4-week-old RPE cultures (Fig. 2), indicating that the substrate supports proper maturation of RPE cells. Next, using a novel device, mechanical stress was added to the RPE monolayer to determine changes in gene and protein expression of major angiogenic and EMT factors. Lastly, an in vitro angiogenesis assay was performed to determine if pro-angiogenic factor production outweighed anti-angiogenic factor production and induced angiogenesis.

Our results showed elevated mRNA expression of angiogenic factors, VEGF, HIF- $1 \alpha$, IL-6, IL-8, ANG2, and anti-angiogenic PEDF (Fig. 3q), and increased protein expression of VEGF, IL-6, IL-8, and ANG2 in mechanically stressed RPE cells (Fig. 3b-p, s, t). ICC results also revealed a remarkable disruption of the actin cytoskeleton in RPE sites with higher VEGF, IL- 6 and IL- 8 expression. The stressed areas of the RPE showed diffusely distributed actin fibers, while in the non-stressed areas, actin was localized in cell-cell junctions (insets in Fig. 3c, h, m). According to the FEA (Fig. 3a), in these areas of impact, strain distribution pattern correlated

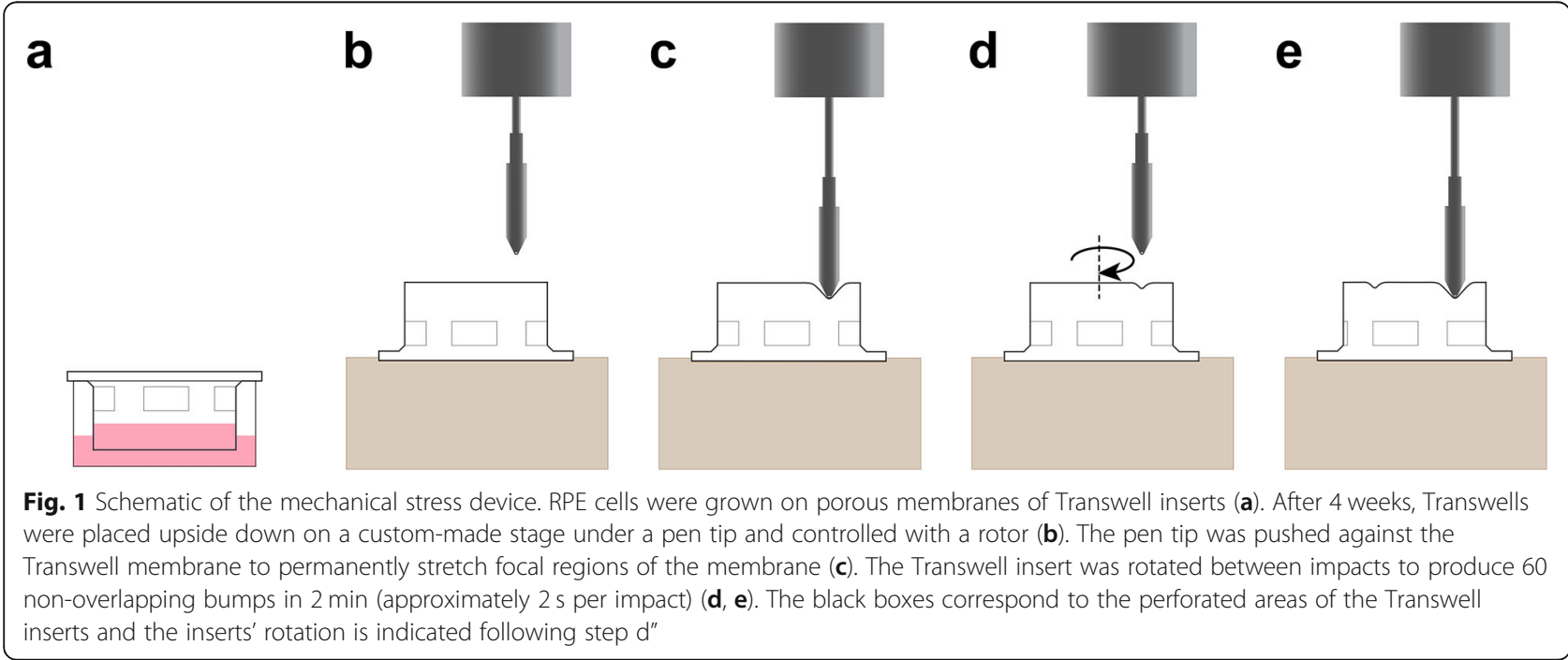



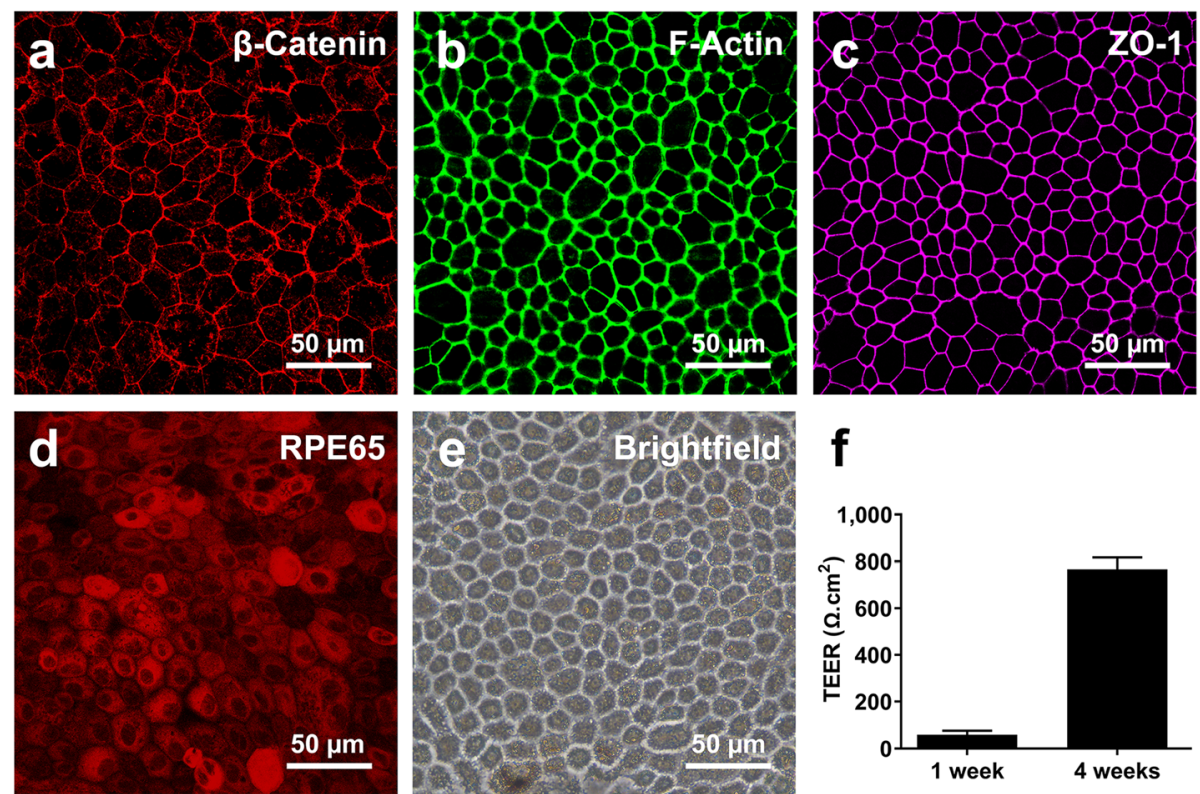

Fig. 2 Characterization of porcine RPE monolayers. ICC results confirm the proper localization of $\beta$-catenin (a), F-actin (b), and ZO-1 (c) and the expression of RPE65 (d) in isolated RPE cells. Brightfield imaging showed the characteristic cobblestone morphology of RPE cells (e). TEER values reached $\sim 767 \Omega . \mathrm{cm}^{2}$ after 4 weeks, indicating maturation of the RPE and establishment of an in vivo-like blood-retinal barrier (f). Error bars represent one standard deviation

with the disruption of actin structure and increased VEGF, IL-6, and IL-8 expression, supporting the hypothesis that the increase in mechanical stress is responsible for elevated angiogenic factor expression.

The increase in IL-8 and VEGF expression (Fig. 3q, s, t) was consistent with previous reports of IL-8 and VEGF induction in human RPE cells after RPE injury $[29,30]$. It has previously been shown that actin polymerization activation induces IL-8 expression and different VEGF isoforms [31, 32] . It is therefore possible that disrupting the actin cytoskeleton activates actin polymerization, leading to the increased expression of IL-8 and different VEGF isoforms. Unlike VEGF121 and $V E G F 165$, VEGF189 was not sensitive to the mechanical stress levels used in our experiments (Fig. 3q). The two soluble VEGF isoforms, VEGF121 and VEGF165, are regulated by low frequency stress while insoluble VEGF189 is more susceptible to high frequency mechanical stress [32]. The single pulse of mechanical stress used in our experiment may be lower than required for VEGF189 mRNA overexpression. Both soluble VEGF isoforms, VEGF121 and VEGF165, have been implicated in in vitro and in vivo neovascularization [33-36]. Hence, their overexpression induced by mechanical stress may contribute to $\mathrm{CNV}$ development.

We also observed an increase in the expression of $H I F-1 \alpha$ in response to mechanical stress (Fig. 3q). HIF$1 \alpha$ is an inducer of VEGF, IL-6, and IL- 8 under hypoxic conditions [37, 38], and its mRNA expression was activated by mechanical stress, suggesting that the mechanisms of mechanical stress-induced VEGF, IL-6, and IL-8 overexpression may be similar to hypoxic conditions. Further investigation is needed to accurately assess the involvement of HIF- $1 \alpha$ in inducing angiogenic factors in RPE cells due to mechanical stress.

Our gene expression results also showed an increase in the expression of TNF- $\alpha$ in response to mechanical stress (Fig. 3q). Mechanical stress can induce oxidative stress in RPE cells, which in turn activates TNF- $\alpha$ transcription [39, 40]. Our qRT-PCR results confirm these previous findings by implicating mechanical stress in inducing TNF- $\alpha$ gene expression. However, ELISA results showed undetectably low levels of TNF- $\alpha$ in RPE supernatants before and after applying mechanical stress, suggesting that the low level of mechanical stress used in this study may not be enough to activate TNF- $\alpha$ protein expression in RPE cells. Further experiments with different mechanical stress levels will elucidate the mechanism of mechanical stress-induced TNF- $\alpha$ expression in RPE cells and explain the lack of TNF- $\alpha$ protein expression despite increased transcription.

The qRT-PCR results showed that mechanical stress promoted an EMT-like phenotype in RPE cells, as demonstrated by an increase in the expression of mesenchymal markers, $C D H 2$, VIM, and FN1, and a decrease in the expression of the RPE-specific RPE65 (Fig. 3r). Previous studies have shown that VEGF, IL-6 and IL- 8 can trigger EMT [15-17]. Our experiments demonstrated 


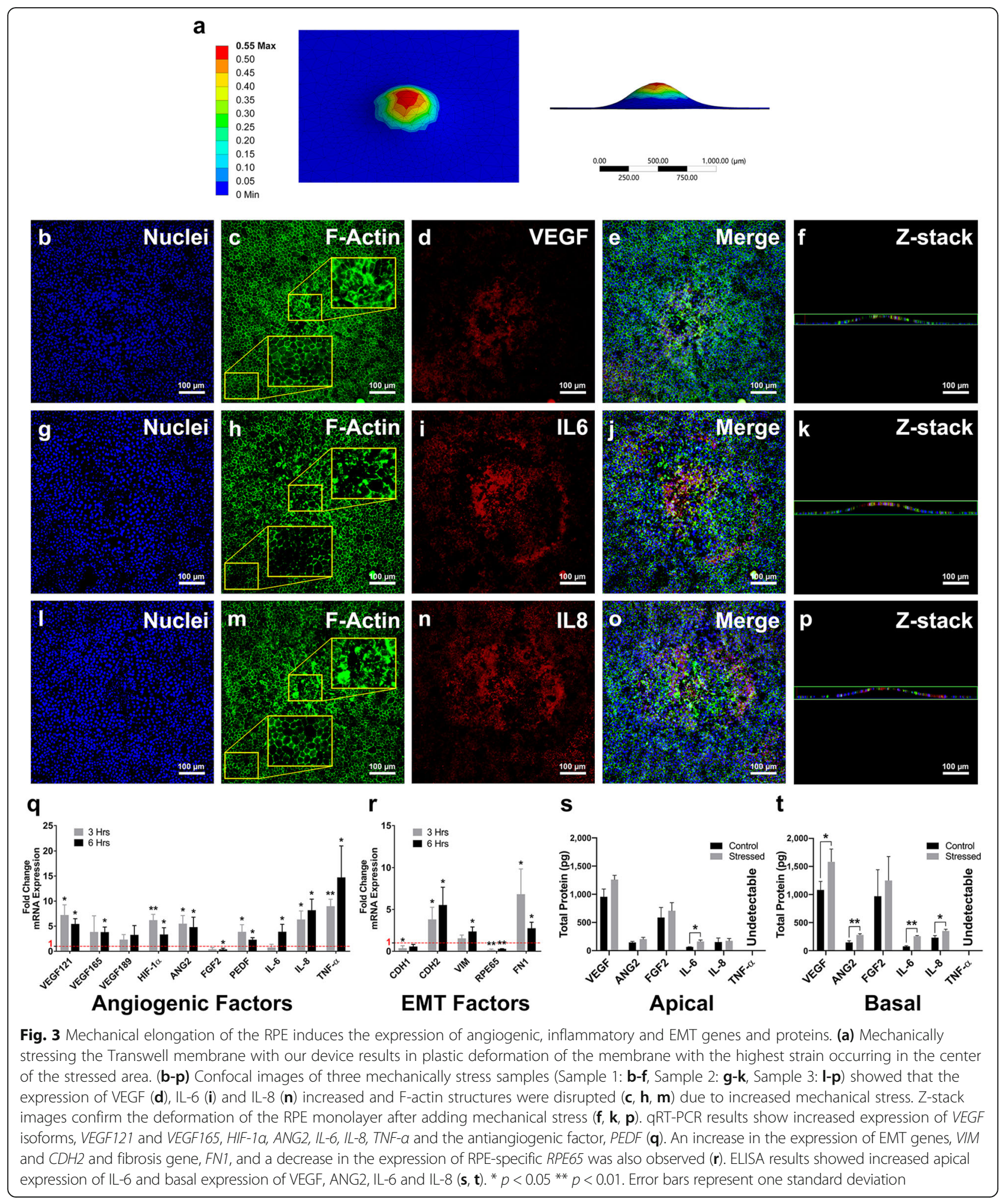

that mechanical stress induced all three of these cytokines, confirming the hypothesis that mechanical stress may induce EMT in the RPE through the induction of EMT promoters during stages of CNV development.
The expression of $P E D F$, a major anti-angiogenic gene, also increased due to mechanical stress (Fig. 3q). Previous work has shown that the balance between VEGF and PEDF must change for choroidal angiogenesis 
initiation [41], The in vitro angiogenesis assay determined if pro-angiogenic protein levels outweigh antiangiogenic factors and promote angiogenesis. The increased angiogenic response of the HUVECs to the mechanically stressed RPE secretome (Fig. 4) suggests that the increase in the PEDF expression was either not sufficient to neutralize the angiogenic activity of the overexpressed angiogenic factors or did not lead to significant protein secretion. However, the higher increase in the angiogenic potential of the apical supernatant compared to that of the basal supernatant (Fig. 4) could be due to the elevated PEDF expression, as the expression of PEDF is mainly basolateral. Further protein expression analyses are needed to better understand the dynamics of the VEGF/PEDF angiogenic switch under mechanical stress. The choroid is adjacent to the basal side of the RPE and our experiments showed that media from both sides of mechanically-stressed RPE cultures increased in vitro angiogenesis (Fig. 4), suggesting that mechanical stress promotes angiogenesis by inducing the overexpression of angiogenic factors. These results deepen our understanding of the role that mechanical stress may play in the initiation and development of $\mathrm{CNV}$ and open potential avenues to more effective therapeutic interventions for neovascularization in AMD.

\section{Conclusions}

In this work, we present a novel method of introducing and understanding mechanical changes in the RPE during early stages of $\mathrm{CNV}$ development and reported, for the first time, that acute mechanical stress induces the expression of angiogenic and EMT factors. In vitro angiogenesis results confirmed the main hypothesis that

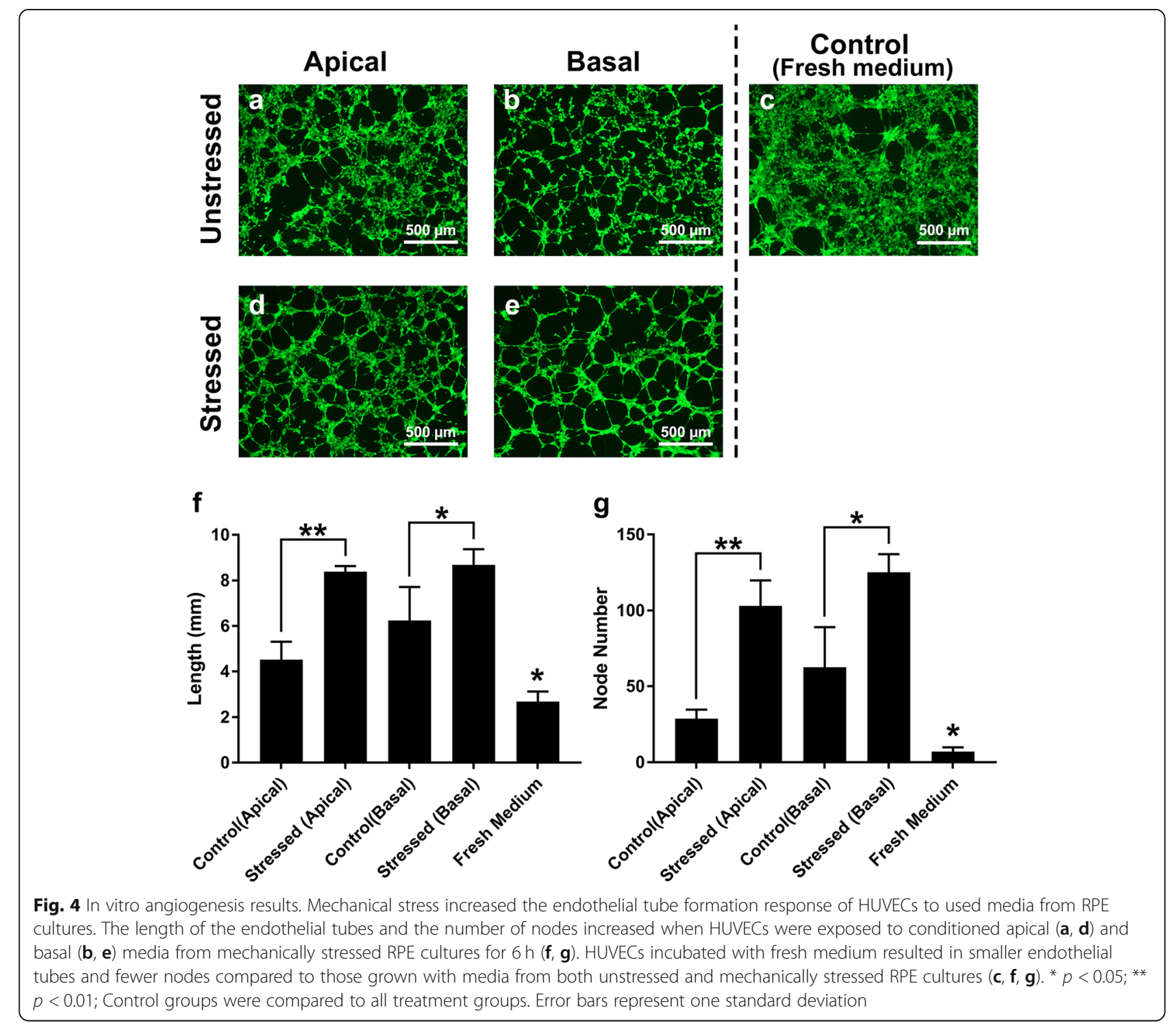


mechanical stress in RPE cells can induce angiogenesis. This result suggests that mechanical stretching of the $\mathrm{RPE}$ accelerates angiogenesis during $\mathrm{CNV}$.

\section{Methods}

To identify the effect of mechanical stress on the expression of angiogenic cytokines and in vitro angiogenesis, we first cultured porcine RPE cells on Transwell membranes for 4 weeks. Using a novel mechanical stress device, the porous membrane of the Transwell inserts was stretched to apply mechanical stress to the overlying RPE cells. Immunocytochemistry, qRT-PCR and enzyme-linked immunosorbent assay were used to measure gene and protein expression of angiogenic cytokines and EMT markers. Finally, an in vitro angiogenesis assay was used to measure the angiogenic potential of the conditioned media from control and mechanically stressed RPE cultures.

\section{RPE isolation and culture}

RPE cells were isolated from locally-sourced pig eyeballs using a previously described method [42]. Isolated cells (passage 0) were cultured on Dulbecco's modification of Eagle medium (DMEM) 1x (Corning, Manassas, VA) supplemented with $10 \%$ premium grade fetal bovine serum (FBS; VWR, Radnor, PA) on $2.4 \mathrm{~mm}$ Transwell inserts (pore size: $0.4 \mu \mathrm{m}$, Corning) and incubated at $37{ }^{\circ} \mathrm{C}, 5 \% \mathrm{CO}_{2}$ in a humidified incubator until the inserts were confluent (approximately $3-5$ days). After reaching confluency, FBS concentration was dropped to $1 \%$ and cells were grown on Transwell inserts for 4 weeks to promote RPE differentiation before the experiments.

\section{Transepithelial electrical resistance (TEER)}

TEER was performed using an EVOM2 voltohmmeter (World Precision Instrument, Sarasota, FL) connected to an ENDOHM-24SNAP measurement chamber (World Precision Instrument). TEER of RPE monolayers was measured after 1 week and 4 weeks of culture on 3 Transwell membranes.

\section{Mechanical stress}

We have previously shown that the plastic properties of the porous polyester Transwell membranes can be permanently stretched to convey mechanical stress to adherent cells [2]. In this work, we fabricated a device to convey controlled and localized stress to RPE monolayers instead. The tip of a ballpoint pen was attached to an in-house reciprocating motion mechanism. A Transwell membrane was placed upside down on a custom stage made from a laser-cut acrylic sheet directly under the pen tip and rotated as the reciprocating motion was engaged to simulate 60 non-overlapping impacts (mechanical stresses) on the membrane over 2 min, creating a single pulse of mechanical stress (Fig. 1). The height of the stage was adjusted so that the tip's pressure created $\sim 10 \%$ strain, mimicking low levels of strain on the RPE during early stages of $\mathrm{CNV}$.

\section{Finite element analysis}

Finite element analysis (FEA) was performed using Ansys V19.1 to evaluate strain distribution on Transwell membranes due to mechanical stress. Plastic strain in the membranes was simulated by pushing a 3D model of the pen tip ( $500 \mu \mathrm{m}$ diameter) on a $10 \mu \mathrm{m}$-thick polyester membrane (same as Transwell membranes) to create $10 \%$ strain.

\section{Immunocytochemistry}

Immunocytochemistry (ICC) was performed on RPE cells grown on mechanically stressed Transwell membranes using anti-VEGF, anti-IL-6, and anti-IL-8 primary antibodies (Santa Cruz Biotechnology, Santa Cruz, CA). RPE65, zonula occludens-1 (ZO-1), and $\beta$-catenin antibodies (Thermo Fisher Scientific, Carlsbad, CA) were also used to evaluate RPE differentiation. F-actin fibers were stained using ActinGreen ${ }^{\text {TM }} 488$ ReadyProbes $^{\text {Th }}$ Reagent (Life Technologies, Eugene, OR) and nuclei were stained with NucBlue Live ReadyProbes stain (Life Technologies). After staining, samples were imaged using an LSM 710 Carl Zeiss confocal microscope (Jena, Germany).

\section{RNA isolation and qRT-PCR}

Three and 6 hours after adding mechanical stress, RPE cells from 8 independent cultures (4 unstressed and 4 mechanically stressed) were lysed directly on the membranes and RNA was isolated using an Illustra RNAspin Mini RNA Isolation kit (GE Healthcare, Chicago, IL). A High Capacity RNA-to-cDNA kit (Thermo Fisher Scientific) was used to obtain cDNA from $1 \mu \mathrm{g}$ of isolated RNA. qRT-PCR was performed using PowerUp ${ }^{\text {tw }}$ SYBR Green Master Mix (Thermo Fisher Scientific) in an Eppendorf RealPlex4 real-time Mastercycler (Hamburg, Germany) to measure the expression levels of three VEGF isoforms (VEGF121, VEGF165 and VEGF189), HIF-1 $\alpha, A N G 2, F G F 2, P E D F, I L-6, I L-8, T N F-\alpha, C D H 1$, $C D H 2$, VIM, RPE65, and FN1. Data were normalized to glyceraldehyde 3-phosphate dehydrogenase (GAPDH) values and fold change expression was calculated using the $2^{-\Delta \Delta \mathrm{Ct}}$ method.

\section{Enzyme-linked immunosorbent assay}

$50 \mu \mathrm{L}$ samples of spent apical and basal media from control and mechanically stressed RPE cultures were collected after $24 \mathrm{~h}$ of applying mechanical stress. The expression of VEGF, ANG2, FGF2, IL-6, IL-8 and TNF$\alpha$ was tested using a multiplex human enzyme-linked 
immunosorbent assay (ELISA) kit according to manufacturer's instructions (Quansys Biosciences, Logan, UT).

\section{Tube formation assay}

Tube formation assays were performed using an in vitro angiogenesis kit (Gibco) according to manufacturer's instructions. Briefly, the wells of a 48-well plate were coated with $100 \mu \mathrm{L}$ of reduced growth factor Geltrex matrix (Gibco) and incubated at $37^{\circ} \mathrm{C}$ for $30 \mathrm{~min}$. Human umbilical vein endothelial cells (HUVECs) were diluted in spent media from unstressed or mechanically stressed RPE cultures to a concentration of $10^{6}$ cells $/ \mathrm{mL}$. $200 \mu \mathrm{L}$ of cell suspensions were seeded on Geltrex matrices and incubated for $6 \mathrm{~h}$ at $37^{\circ} \mathrm{C}$ in a humidified incubator with $5 \% \mathrm{CO}_{2}$ to induce endothelial tube formation. Next, HUVECs were stained with Calcein AM dye (Thermo Fisher Scientific) and imaged using an Eclipse TS100 fluorescence microscope (Nikon Instrument Inc., Melville, NY). Tube length and node numbers were quantified using the ridge detection plugin for ImageJ software [43, 44].

\section{Statistical analysis}

The data are presented as the mean \pm standard deviation (SD) of at least three independent experiments. Comparisons between two groups were analyzed using twotailed Student's $t$-test and $p$-values were adjusted using the Benjamini-Hochberg method. $P<0.05$ was considered statistically significant.

\section{Acknowledgements}

The authors thank Cynthia Hanson and Eryn Hanson for their assistance in editing the manuscript.

\section{Authors' contributions \\ FF designed the study, collected data, performed analyses, and wrote the manuscript. AA and SO collected data, performed analyses, and wrote the manuscript. EV is the corresponding author, designed the study, and provided equipment, funding, and oversight to the project. In addition, EV edited and approved the manuscript for publication. The author(s) read and approved the final manuscript.}

\section{Funding}

This work was supported by a National Eye Institute of the National Institutes of Health Grant R15EY028732, a Career Starter Grant from the Knights Templar Eye Foundation, a Ralph E. Powe Junior Faculty Award from the Oak Ridge Associated Universities (ORAU), Utah State University's Office of Graduate Studies, and Utah State University's College of Engineering.

\section{Availability of data and materials}

The datasets generated and analyzed during the current study are available from the corresponding author on reasonable request.

\section{Ethics approval and consent to participate} N/A.

\section{Consent for publication}

N/A

\section{Competing interests}

The authors declare that they have no competing interests.

\section{Author details}

'Department of Biological Engineering, Utah State University, 4105 Old Main Hill, Logan, UT 84322, USA. Present address: Neural Stem Cell Institute, Rensselaer, NY 12144, USA. ${ }^{3}$ Present address: Department of Animal Sciences, Yasouj University, Yasouj 75918-74934, Iran. ${ }^{4}$ Division of General Medicine, Columbia University Medical Center, New York, NY 10032, USA.

Received: 9 January 2020 Accepted: 24 March 2020

Published online: 25 April 2020

\section{References}

1. Wu S, Lu Q, Wang N, Zhang J, Liu Q, Gao M, et al. Cyclic stretch inducedretinal pigment epithelial cell apoptosis and cytokine changes. BMC Ophthalmol. 2017;17:208.

2. Farjood F, Vargis E. Novel devices for studying acute and chronic mechanical stress in retinal pigment epithelial cells. Lab Chip. 2018;18:3413-24.

3. Notomi S, Hisatomi T, Murakami Y, Terasaki H, Sonoda S, Asato R, et al. Dynamic increase in extracellular ATP accelerates photoreceptor cell apoptosis via ligation of P2RX7 in subretinal hemorrhage. PLoS One. 2013;8: e53338.

4. Yehoshua Z, Wang F, Rosenfeld PJ, Penha FM, Feuer WJ, Gregori G. Natural history of drusen morphology in age-related macular degeneration using spectral domain optical coherence tomography. Ophthalmology. 2011;118: 2434-41.

5. Stanescu-Segall D, Balta F, Jackson TL. Submacular hemorrhage in neovascular age-related macular degeneration: a synthesis of the literature. Surv Ophthalmol. 2016;61:18-32.

6. Spilsbury K, Garrett KL, Shen W-Y, Constable IJ, Rakoczy PE. Overexpression of vascular endothelial growth factor (VEGF) in the retinal pigment epithelium leads to the development of choroidal neovascularization. Am J Pathol. 2000;157:135-44.

7. Martin G, Schlunck G, Hansen LL, Agostini HT. Differential expression of angioregulatory factors in normal and CNV-derived human retinal pigment epithelium. Graefes Arch Clin Exp Ophthalmol. 2004;242:321-6.

8. Stahl A, Paschek L, Martin G, Feltgen N, Hansen LL, Agostini HT. Combinatory inhibition of VEGF and FGF2 is superior to solitary VEGF inhibition in an in vitro model of RPE-induced angiogenesis. Graefes Arch Clin Exp Ophthalmol Albrecht Von Graefes Arch Klin Exp Ophthalmol. 2009; 247:767-73

9. Jasielska M, Semkova I, Shi X, Schmidt K, Karagiannis D, Kokkinou D, et al. Differential role of tumor necrosis factor (TNF)-a receptors in the development of choroidal neovascularization. Invest Ophthalmol Vis Sci. 2010;51:3874-83.

10. Roh Ml, Kim HS, Song JH, Lim JB, Koh HJ, Kwon OW. Concentration of cytokines in the aqueous humor of patients with naive, recurrent and regressed CNV associated with amd after bevacizumab treatment. Retina Phila Pa. 2009;29:523-9.

11. Izumi-Nagai K, Nagai N, Ozawa Y, Mihara M, Ohsugi Y, Kurihara T, et al. Interleukin-6 receptor-mediated activation of signal transducer and activator of transcription-3 (STAT3) promotes choroidal neovascularization. Am J Pathol. 2007;170:2149-58.

12. Lai K, Luo C, Zhang X, Ye P, Zhang Y, He J, et al. Regulation of angiogenin expression and epithelial-mesenchymal transition by HIF-1a signaling in hypoxic retinal pigment epithelial cells. Biochim Biophys Acta (BBA) - Mol Basis Dis. 1862;2016:1594-607.

13. Hirasawa M, Noda K, Noda S, Suzuki M, Ozawa Y, Shinoda K, et al. Transcriptional factors associated with epithelial-mesenchymal transition in choroidal neovascularization. Mol Vis. 2011;17:1222-30.

14. Takahashi E, Nagano O, Ishimoto T, Yae T, Suzuki Y, Shinoda T, et al. Tumor necrosis factor-a regulates transforming growth factor- $\beta$-dependent epithelial-mesenchymal transition by promoting hyaluronan-CD44-moesin interaction. J Biol Chem. 2010;285:4060-73.

15. Gonzalez-Moreno O, Lecanda J, Green JE, Segura V, Catena R, Serrano D, et al. VEGF elicits epithelial-mesenchymal transition (EMT) in prostate intraepithelial neoplasia (PIN)-like cells via an autocrine loop. Exp Cell Res. 2010;316:554-67.

16. Zhou J, Zhang C, Pan J, Chen L, Qi S-T. Interleukin-6 induces an epithelialmesenchymal transition phenotype in human adamantinomatous craniopharyngioma cells and promotes tumor cell migration. Mol Med Rep. 2017;15:4123-31. 
17. Zhou N, Lu F, Liu C, Xu K, Huang J, Yu D, et al. IL-8 induces the epithelialmesenchymal transition of renal cell carcinoma cells through the activation of AKT signaling. Oncol Lett. 2016;12:1915-20.

18. Skovseth DK, Küchler AM, Haraldsen G. The HUVEC/Matrigel assay. In: Sioud M, editor. Target Discov valid rev Protoc Vol 1 Emerg Strateg targets biomark Discov. Totowa: Humana Press; 2007. p. 253-68. [Cited 2020 Mar 4. https://doi.org/10.1385/1-59745-165-7:253.

19. Chen Z, Htay A, Santos WD, Gillies GT, Fillmore HL, Sholley MM, et al. In vitro angiogenesis by human umbilical vein endothelial cells (HUVEC) induced by three-dimensional co-culture with glioblastoma cells. J NeuroOncol. 2009;92:121-8.

20. Farjood F, Vargis E. Physical disruption of cell-cell contact induces VEGF expression in RPE cells. Mol Vis. 2017;23:431-46.

21. Yoshino H, Morita I, Murota S, Ishikawa I. Mechanical stress induces production of angiogenic regulators in cultured human gingival and periodontal ligament fibroblasts. J Periodontal Res. 2003;38:405-10.

22. Zhu Z, Gan X, Fan H, Yu H. Mechanical stretch endows mesenchymal stem cells stronger angiogenic and anti-apoptotic capacities via NFKB activation. Biochem Biophys Res Commun. 2015;468:601-5.

23. David M, Cullen JP, Cahill PA, Redmond Eileen M. Cyclic Strain Regulates the Notch/CBF-1 Signaling Pathway in Endothelial Cells. Arterioscler Thromb Vasc Biol. 2007;27:1289-96.

24. Seko $Y$, Seko $Y$, Fujikura H, Pang J, Tokoro T, Shimokawa H. Induction of vascular endothelial growth factor after application of mechanical stress to retinal pigment epithelium of the rat in vitro. Invest Ophthalmol Vis Sci. 1999:40:3287-91.

25. Ablonczy Z, Dahrouj M, Tang PH, Liu Y, Sambamurti K, Marmorstein AD, et al. Human retinal pigment epithelium cells as functional models for the RPE in vivo. Invest Ophthalmol Vis Sci. 2011;52:8614-20

26. Wiencke AK, Kiilgaard JF, Nicolini J, Bundgaard M, Röpke C, La Cour M. Growth of cultured porcine retinal pigment epithelial cells. Acta Ophthalmol Scand. 2003:81:170-6.

27. Ablonczy Z, Crosson CE. VEGF modulation of retinal pigment epithelium resistance. Exp Eye Res. 2007:85:762-71.

28. Dithmer M, Fuchs S, Shi Y, Schmidt H, Richert E, Roider J, et al. Fucoidan reduces secretion and expression of vascular endothelial growth factor in the retinal pigment epithelium and reduces angiogenesis in vitro. PLoS One. 2014:9:e89150.

29. Yoshida A, Elner SG, Bian Z-M, Elner VM. Induction of interleukin-8 in human retinal pigment epithelial cells after denuding injury. Br J Ophthalmol. 2001; 85:872-6.

30. Greene WA, Burke TA, Por ED, Kaini RR, Wang H-C. Secretion profile of induced pluripotent stem cell-derived retinal pigment epithelium during wound HealingStem cell-derived RPE profile during wound healing. Invest Ophthalmol Vis Sci. 2016:57:4428-41.

31. Gao M, Wu S, Ji J, Zhang J, Liu Q, Yue Y, et al. The influence of actin depolymerization induced by Cytochalasin D and mechanical stretch on interleukin-8 expression and JNK phosphorylation levels in human retinal pigment epithelial cells. BMC Ophthalmol. 2017;17:43.

32. Faure $\mathrm{C}$, Linossier M-T, Malaval L, Lafage-Proust M-H, Peyroche S, Vico L, et al. Mechanical signals modulated vascular endothelial growth factor-a (VEGF-A) alternative splicing in osteoblastic cells through actin polymerisation. Bone. 2008;42:1092-101.

33. Browning AC, Dua HS, Amoaku WM. The effects of growth factors on the proliferation and in vitro angiogenesis of human macular inner choroidal endothelial cells. Br J Ophthalmol. 2008;92:1003-8.

34. Wang F, Rendahl KG, Manning WC, Quiroz D, Coyne M, Miller SS. AAVmediated expression of vascular endothelial growth factor induces Choroidal neovascularization in rat. Invest Ophthalmol Vis Sci. 2003;44:781-90.

35. Byun J, Heard JM, Huh JE, Park SJ, Jung EA, Jeong JO, et al. Efficient expression of the vascular endothelial growth factor gene in vitro and in vivo, using an adeno-associated virus vector. J Mol Cell Cardiol. 2001;33:295-305.

36. Su H, Lu R, Kan YW. Adeno-associated viral vector-mediated vascular endothelial growth factor gene transfer induces neovascular formation in ischemic heart. Proc Natl Acad Sci U S A. 2000;97:13801-6.

37. Arjamaa O, Aaltonen V, Piippo N, Csont T, Petrovski G, Kaarniranta K, et al. Hypoxia and inflammation in the release of VEGF and interleukins from human retinal pigment epithelial cells. Graefes Arch Clin Exp Ophthalmol Albrecht Von Graefes Arch Klin Exp Ophthalmol. 2017;255:1757-62.

38. Cane $G$, Ginouvès $A$, Marchetti $S$, Buscà R, Pouysségur J, Berra E, et al. HIF1alpha mediates the induction of IL-8 and VEGF expression on infection with Afa/Dr diffusely adhering E. coli and promotes EMT-like behaviour. Cell Microbiol. 2010:12:640-53.

39. Liang X, Wang Z, Gao M, Wu S, Zhang J, Liu Q, et al. Cyclic stretch induced oxidative stress by mitochondrial and NADPH oxidase in retinal pigment epithelial cells. BMC Ophthalmol. 2019;19:79.

40. Hanus J, Zhang H, Wang Z, Liu Q, Zhou Q, Wang S. Induction of necrotic cell death by oxidative stress in retinal pigment epithelial cells. Cell Death Dis. 2013;4:e965.

41. Tong J-P, Yao Y-F. Contribution of VEGF and PEDF to choroidal angiogenesis: a need for balanced expressions. Clin Biochem. 2006;39:267-76.

42. Toops KA, Tan LX, Lakkaraju A. A detailed three-step protocol for live imaging of intracellular traffic in polarized primary porcine RPE monolayers. Exp Eye Res. 2014;124:74-85.

43. Steger C. An unbiased detector of curvilinear structures. IEEE Trans Pattern Anal Mach Intell. 1998:20:113-25.

44. Thorsten Wagner, Mark Hiner, xraynaud. thorstenwagner/ij-ridgedetection: Ridge Detection 1.4.0. Zenodo; 2017 [Cited 2020 Mar 4]. Available from: https://zenodo.org/record/845874\#.XmBgMJNKiuU.

\section{Publisher's Note}

Springer Nature remains neutral with regard to jurisdictional claims in published maps and institutional affiliations.
Ready to submit your research? Choose BMC and benefit from:

- fast, convenient online submission

- thorough peer review by experienced researchers in your field

- rapid publication on acceptance

- support for research data, including large and complex data types

- gold Open Access which fosters wider collaboration and increased citations

- maximum visibility for your research: over $100 \mathrm{M}$ website views per year

At $\mathrm{BMC}$, research is always in progress.

Learn more biomedcentral.com/submissions 